\title{
Efficacy and safety of topical BAK-free travoprost $0.004 \%$ versus BAK- preserved travoprost $0.004 \%$ in the treatment of primary open angle glaucoma: a comparative study at a tertiary care hospital
}

\author{
Jayanthi C. R. ${ }^{1}$, Divyashree R. N. ${ }^{1 *}$, Sujatha B. L. ${ }^{2}$
}

\begin{abstract}
${ }^{1}$ Department of Pharmacology, Bangalore Medical College and Research Institute, Bengaluru, Karnataka, India

${ }^{2}$ Department of Ophthalmology, Minto Ophthalmic Hospital, Bangalore Medical College and Research Institute, Bengaluru, Karnataka, India
\end{abstract}

Received: 27 June 2017 Accepted: 24 July 2017

\section{*Correspondence to: Dr. Divyashree R. N., Email: drdivyavinay@ yahoo.co.in}

Copyright: (c) the author(s), publisher and licensee Medip Academy. This is an openaccess article distributed under the terms of the Creative Commons Attribution NonCommercial License, which permits unrestricted noncommercial use, distribution, and reproduction in any medium, provided the original work is properly cited.

\begin{abstract}
Background: Prostaglandin analogues (PGAs) reduce intraocular pressure (IOP) in patients with primary open-angle glaucoma (POAG); however, these medications may affect the ocular surface and elicit ocular discomfort when preserved with benzalkonium chloride (BAK). Hence the above study was taken to evaluate the benefit of BAK-free formulations of travoprost. The objectives of the study were to compare the efficacy, safety of topical BAK-free travoprost $0.004 \%$ versus BAK-preserved travoprost $0.004 \%$ in patients with primary open angle glaucoma.
\end{abstract}

Methods: 40 patients with POAG who fulfilled the inclusion /exclusion criteria were randomised into two groups of 20 each to receive BAK-free travoprost $0.004 \%$ or BAK-preserved travoprost once daily in the evening. Efficacy was measured in terms of reduction in IOP monitored at 4,8 and 12 weeks from baseline. Ocular surface disease index (OSDI) questionnaire was used to assess the ocular surface symptoms. Safety was assessed by monitoring treatment emergent adverse drug reactions (ADRs).

Results: Both the study medications were effective in reducing IOP when compared to baseline. Mean IOP reduction from baseline to week 12 was $11 \pm 3 \mathrm{mmHg}(\mathrm{p}<0.001), 10.78 \pm 3.01 \mathrm{mmHg},(\mathrm{p}<0.001)$ in BAK-free travoprost and BAK-preserved travoprost groups respectively. Both produced equivalent reductions in IOP at the end of $4(7.89 \pm 1.82$ vs $7.63 \pm 2.83, p=0.72), 8(9.94 \pm 2.75$ vs10.05 $\pm 2.75, \mathrm{p}=0.90)$, and 12 weeks $(11 \pm 3$ vs10.78 $\pm 3.01, \mathrm{p}=0.82)$. BAK-free travoprost demonstrated significantly lower OSDI scores $(15.10 \pm 3.60)$ compared to BAK- preserved travoprost $(23.47 \pm 7.10)$ at 12 weeks $(\mathrm{p}<0.0001)$. There was no significant difference in occurrence of conjunctival hyperaemia between the study drugs $\left(\chi^{2}=0, \mathrm{df}=1, \mathrm{p}=1\right)$ and BAK-free travoprost was well tolerated. Conclusions: BAK-free and BAK-preserved travoprost significantly reduced IOP at 12 weeks. But, BAK- free travoprost produced significantly less ocular surface symptoms as compared to BAK- preserved travoprost. Hence it could be a favourable option in POAG patients with ocular surface disease symptoms.

Keywords: BAK, BAK-free travoprost, Intraocular pressure, OSDI, Ocular surface disease index, Primary open angle glaucoma

\section{INTRODUCTION}

Primary open-angle glaucoma is an optic neuropathy characterized by loss of retinal ganglion cells and their axons, resulting in progressive loss of the peripheral visual field. Axonal loss is manifested as thinning of the optic nerve head's neuro-retinal rim, producing the characteristic cupping of the nerve. ${ }^{1}$ If untreated or inadequately treated, glaucoma can lead to blindness. POAG, which accounts for the majority of disease cases, primarily results from impaired or suboptimal drainage of aqueous humour out of the eye via the trabecular meshwork and/or uveoscleral pathways. ${ }^{2}$ It has been estimated that by 2010, almost 45 million people will have POAG worldwide, and by 2020 this number is expected to 
increase to 58.5 million. In India, glaucoma is responsible for approximately $5.8 \%$ of blindness. ${ }^{3}$ The central role of IOP reduction in decreasing the risk of development or progression of POAG has been borne out in several landmark randomized controlled trials and thus drugs that reduce IOP have the potential to prevent or delay optic nerve damage and preserve vision.

PGAs are shown to be superior to older drugs in both efficacy and tolerability. Travoprost being one of the member of PGAs class, lowers IOP by enhancing the egress of aqueous humour through both the uveoscleral and trabecular outflow channels. ${ }^{4}$

Long term use of topical drugs has clearly been shown to induce toxic immunolopathological changes in the ocular surface. It has been reported by Fechtner, et al that prevalence of ocular surface disease (OSD) symptoms in glaucoma patients is $48.4 \%$ and the severity of OSD symptoms increases with the number of medications used. Though PG analogues produce much fewer side effects, the adherence and compliance to medication regimens are surprisingly lower than expected. A commonly cited reason is the ocular irritation and inflammation with these medications worsening the ocular surface symptoms. Much of this inflammation can be attributed to the preservative, BAK. ${ }^{5-7}$

BAK is the most commonly used preservative in ophthalmic preparations with a high affinity for membrane proteins and may accumulate in ocular tissues, inducing cell toxicity and/or cell death in a dose-dependent manner. It has also been shown to damage ocular tissue by inducing apoptosis and increasing the concentrations of inflammatory markers. ${ }^{5-7}$

Travoprost BAK-free ophthalmic solution is the first commercially available preparation of a prostaglandin analogue preserved without BAK ${ }^{8}$ It contains an ionic buffered preservative system, sofZia ${ }^{\mathrm{TM}}$ which is an ionic, buffered formula composed of zinc, borate, propylene glycol, and sorbitol. SofZia is effective in microbicidal activity for up to $99 \%$ of microorganisms after an 8-day incubation, and it meets the antimicrobial criteria of the United States Pharmacopeia. ${ }^{9}$ It is said to have equivalent IOP lowering capacity as BAK-preserved travoprost and less conjunctival hyperaemia.

The purpose of the present study was to examine the efficacy, safety of travoprost BAK-free ophthalmic solution compared to BAK-preserved travoprost as there is a paucity of data evaluating the clinical benefit of eliminating BAK from prostaglandin analogue therapy amongst Indian patients.

\section{METHODS}

This was an open label, randomized prospective study conducted between May 2016-October 2016 in the Regional Institute of Ophthalmology, Minto Hospital attached to Bangalore Medical College and Research Institute, Bengaluru.

After obtaining institutional ethics committee clearance and written informed consent, the out-patients at the glaucoma clinic of either sex aged 18 years and above, diagnosed to be suffering from primary open angle glaucoma with mean ( 2 readings) IOP of $\geq 21 \mathrm{mmHg}$ at the baseline visit with ocular surface symptoms were enrolled in the study.

Patients suffering from amblyopia, legal blindness (6/60 or less) in either eye, acute angle closure glaucoma, optic nerve disease, ocular infection or inflammation within the previous 3 months and anticipated use of topical or systemic steroids were excluded. Patients with severe trauma, any systemic contraindications or hypersensitivity to study medications were also excluded from the study. For the assessment of ocular surface symptoms supplementary exclusion criteria were as follows: OSD that had previously been treated with punctal plugs, punctal cautery, topical cyclosporine A, suspected or diagnosed Sjögren's syndrome; prior corneal surgery (including keratorefractive surgery) within the previous one year; presence or history of clinically significant blepharitis within the previous two years; seasonal ocular allergies expected within the study period; and any contact lens wear or corticosteroid use within the 30 days before the screening visit.

A total of 40 patients were recruited and randomized in a 1:1 ratio into two groups of 20 each using computer generated randomization sequence (www.random.org/sequences) to receive 1 drop of either BAK-free travoprost $0.004 \%$ or BAK-preserved travoprost $0.004 \%$ eye drops once daily in the evening.

Demographic data, ocular history, medical history, concomitant medications and details of general, systemic and ophthalmological examination were recorded in the study proforma at baseline visit (visit 1). Follow-up was done at 4 weeks (visit 2), 8 weeks (visit 3) and 12 weeks (visit 4) after administering the study drugs. At follow-up visits pulse rate, blood pressure, IOP, slit lamp examination findings and visual acuity were recorded. When both eyes fulfilled the eligibility criteria, both were regarded as study eyes and IOP was measured in each eye at the subsequent follow-up visits. IOP was measured with Goldmann applanation tonometer and mean of 2 readings was taken at each of the visits. Concomitant medications were recorded. The Ocular Surface Disease Index (OSDI), a validated questionnaire, was used to examine the prevalence of dry eye/OSD complaints in glaucoma patients. ${ }^{10,11}$ It was administered to the patients by the investigator at baseline and at week 12 and a check was placed in the box that corresponded to their symptoms. The individual answers corresponded to a specific value; all of the time $=4$, most of the time $=3$, half of the time $=2$, some of the time $=1$, and none of the time $=0$. 
Total OSDI score $=\underline{(\text { Sum of score for all questions answered }) \times(25)}$ (Total \# of questions answered)

The total OSDI score was used to classify the severity of OSD. The classification of normal, mild, moderate, or severe OSD was determined on a scale from 0 to 100 . OSD severity was classified as follows: normal (0-12), mild (1322), moderate (23-32), and severe (33-00). Global Evaluation of Overall Tolerability (GEOT) was assessed at 12 weeks. New systemic medications were permitted provided they had no known effect on the IOP. Adverse drug reactions were recorded and graded according to severity as mild (awareness of sign or symptom, but easily tolerated), moderate (enough discomfort to cause interference with usual activity) and severe (incapacitating with inability to work or do usual activity) in the CDSCO (Central Drugs Standard Control Organization) ADR reporting form.

\section{Sample size calculation}

Sample size was estimated as 19 subjects in each group (using two sample mean: equality) with a difference in the mean being $1.1 \mathrm{mmHg}$ and common standard deviation being 1.2 which was obtained from previous studies. Alpha error was set at $5 \%$ and power of the study at $80 \%$. For better computation of results a sample size of 40 was included in the study.

\section{Statistical analysis}

The data collected was tabulated and analyzed using mean and standard deviation. Continuous variables were compared within the group using repeated measures ANOVA and between the groups using unpaired t-test. Categorical data was expressed as percentages/proportions and Chi-square test was done to compare the categorical variables. Statistical significance was defined as a $p$ value of $<0.05$. Analyses were performed using Vassar Stats.

\section{RESULTS}

56 subjects were screened for inclusion in the study of whom 40 subjects who met the inclusion criteria and gave written informed consent to participate in the study were enrolled in the study. The flow chart of recruitment, randomization and follow up is depicted in Figure 1.

\section{Demographic characteristics}

Demographic profile of the patients included in the study is represented in Table 1. The mean age in the BAK-free travoprost group was $62.84 \pm 5.18$ years and $62.05 \pm 8.59$ years in the BAK-preserved travoprost group $(\mathrm{p}=0.73)$. There were $12(60 \%)$ male and $08(40 \%)$ female patients in the BAK-free travoprost group and $10(50 \%)$ male and $10(50 \%)$ female patients in the BAK- preserved group $\left(\chi^{2}\right.$ $=0.1, \mathrm{df}=1, \mathrm{p}=0.75)$. There was no significant difference with respect to demographic characteristics at baseline between groups.

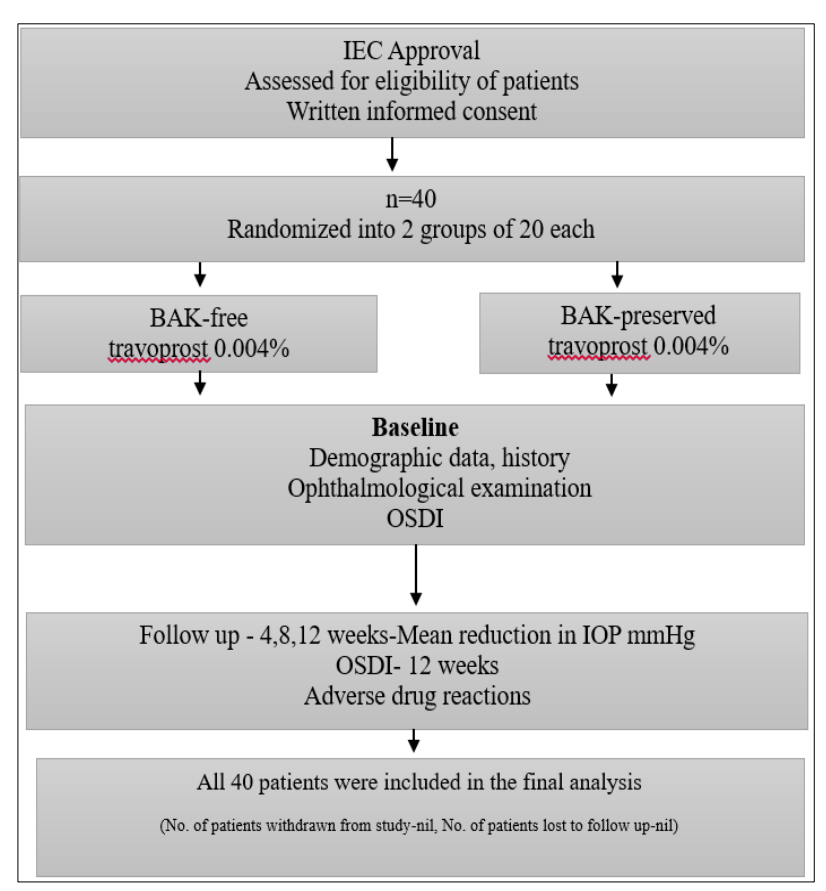

Figure 1: Recruitment, randomisation and follow up.

Table 1: Demographic characteristics of the study population.

\begin{tabular}{|c|c|c|c|}
\hline Parameters & $\begin{array}{l}\text { Group } 1 \\
\text { BAK-free } \\
\text { travoprost } \\
(\mathbf{n}=20)\end{array}$ & $\begin{array}{l}\text { Group } 2 \\
\text { BAK- } \\
\text { preserved } \\
\text { travoprost } \\
(\mathrm{n}=20)\end{array}$ & $\begin{array}{l}\mathbf{p} \\
\text { value }\end{array}$ \\
\hline \multicolumn{4}{|l|}{ Age } \\
\hline $41-60$ years & 04 & 08 & \multirow{2}{*}{$0.73 *$} \\
\hline$\geq 60$ years & 16 & 12 & \\
\hline \multicolumn{4}{|l|}{ Gender } \\
\hline Male & $12(60 \%)$ & $10(50 \%)$ & \multirow{2}{*}{$0.75 * *$} \\
\hline Female & $08(40 \%)$ & $10(50 \%)$ & \\
\hline \multicolumn{4}{|l|}{ Habits } \\
\hline Smoking & $05(25 \%)$ & $07(35 \%)$ & \multirow{2}{*}{$0.33 * *$} \\
\hline Alcohol & $01(5 \%)$ & $03(15 \%)$ & \\
\hline \multicolumn{4}{|l|}{ Comorbidities } \\
\hline Nil & $12(60 \%)$ & $10(50 \%)$ & \multirow{4}{*}{$0.75 * *$} \\
\hline DM & $04(20 \%)$ & $06(30 \%)$ & \\
\hline HTN & $03(15 \%)$ & $02(10 \%)$ & \\
\hline $\mathrm{DM}+\mathrm{HTN}$ & $01(5 \%)$ & $02(10 \%)$ & \\
\hline \multicolumn{4}{|l|}{ OSDI } \\
\hline Normal (0-12) & - & - & \multirow{4}{*}{$0.24 *$} \\
\hline Mild (13-22) & $11(55 \%)$ & $12(60 \%)$ & \\
\hline $\begin{array}{l}\text { Moderate } \\
(23-32)\end{array}$ & $08(40 \%)$ & $08(40 \%)$ & \\
\hline Severe (33-100) & $01(5 \%)$ & - & \\
\hline
\end{tabular}

* Data analysed using unpaired t test, **Data analysed using Chisquare, $\mathrm{p}<0.05$ was taken as statistical significance

\section{Mean reduction in $I O P$}

Both BAK-free travoprost and BAK-preserved travoprost 
free travoprost and BAK preserved travoprost effectively reduced IOP at 12 weeks compared to baseline (ANOVA, $\mathrm{p}<0.0001$, Figure 2).

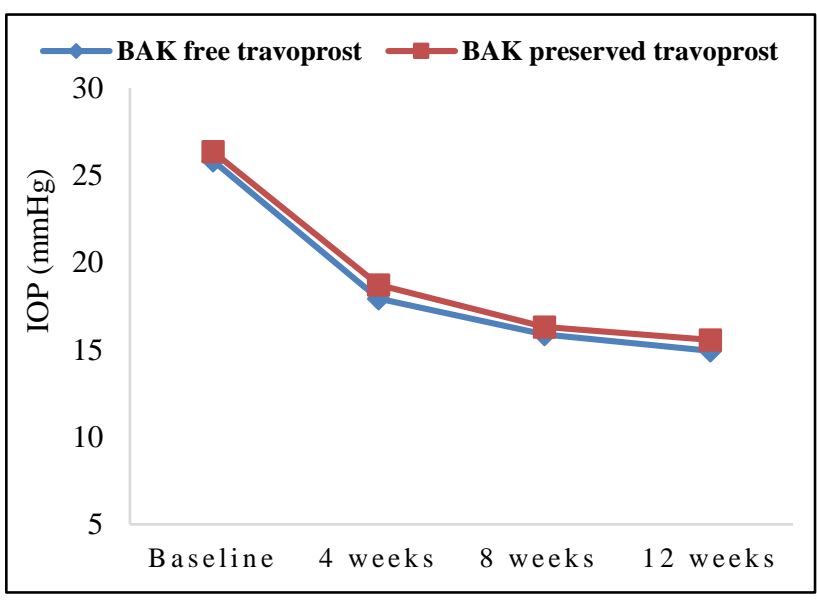

Figure 2: Temporal depiction of mean IOP in the study groups.

There was no statistically significant reduction in IOP
[BAK-free travoprost $(12.78 \pm 2.67 \mathrm{mmHg})$ and BAKpreserved travoprost $(12.47 \pm 3.11 \mathrm{mmHg}, \mathrm{p}=0.73)]$ at week 12 from baseline between the two groups (Figure 3).

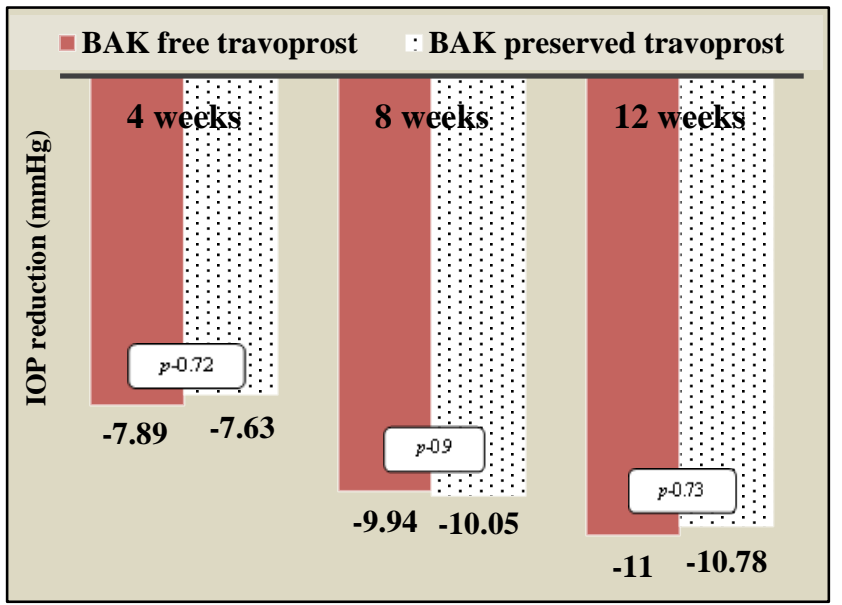

Figure 3: Reduction in IOP at 4, 8 and 12 weeks from baseline.

Table 2: Mean IOP at each visit in the BAK-free travoprost and BAK-preserved travoprost groups.

\begin{tabular}{|c|c|c|c|c|c|}
\hline $\begin{array}{l}\text { Group } \\
\mathbf{n}=\mathbf{2 0} \text { in each group }\end{array}$ & $\begin{array}{l}\text { Baseline } \\
\text { (mmHg) }\end{array}$ & $\begin{array}{l}\text { Week } 4 \\
(\mathrm{mmHg})\end{array}$ & $\begin{array}{l}\text { Week } 8 \\
(\mathrm{mmHg})\end{array}$ & $\begin{array}{l}\text { Week } 12 \\
(\mathrm{mmHg}) \\
\end{array}$ & p value \\
\hline BAK-free travoprost (Mean \pm SD) & $25.84 \pm 3.13$ & $17.94 \pm 2.22$ & $15.89 \pm 1.69$ & $14.94 \pm 1.22$ & $<0.0001^{* \$}$ \\
\hline BAK-preserved travoprost (Mean \pm SD) & $26.36 \pm 2.79$ & $18.73 \pm 2.40$ & $16.31 \pm 2.33$ & $15.57 \pm 2.45$ & $<0.0001^{* \$}$ \\
\hline $\begin{array}{l}\mathrm{p} \text { value - intergroup comparison at each } \\
\text { follow up visit }\end{array}$ & $0.58^{\dagger}$ & $0.72^{\dagger}$ & $0.9^{\dagger}$ & $0.73^{\dagger}$ & \\
\hline
\end{tabular}

*-Data analysed using repeated measures ANOVA, \$statistically significant

$\dagger$ - Data analysed using unpaired test

\section{$\square$ BAK free travoprost $=$ BAK preserved travoprost}

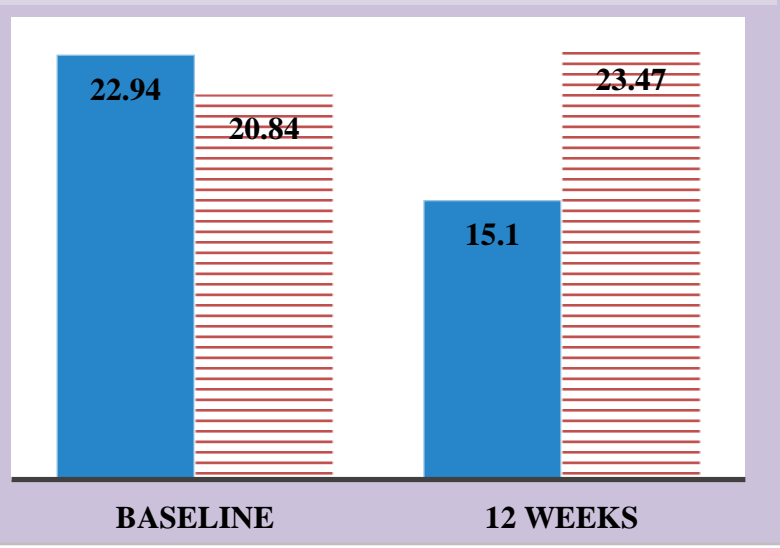

Figure 4: Mean change in OSDI scores between groups.

\section{Ocular surface disease symptoms}

In the above study all the patients at baseline had symptoms of ocular surface disease and the distribution of severity in represented in Table 1 . The baseline OSDI scores was $22.94 \pm 5.90$ and $20.84 \pm 5.70$ in the BAK-free travoprost group and BAK-preserved travoprost group respectively with no statistically significant difference $(\mathrm{p}=0.23)$. The mean change in OSDI scores between the baseline and the 12-week follow-up visit was assessed. On examination for the overall cohort of patients, BAK-free travoprost demonstrated significantly lower OSDI scores (15.10 \pm 3.60$)$ compared to BAK preserved travoprost $(23.47 \pm 7.10)$ at 12 weeks which was significant ( $\mathrm{p}<0.0001$, Figure 4$)$. The use of BAK-free travoprost for 12 weeks significantly reduced the category of severity to the next lower level (moderate to mild, mild to normal). Over $90 \%(n=18)$ of patients experienced a decrease in their OSDI score by at least 1 level of severity after 12 weeks of treatment with BAK-free travoprost. In the BAKpreserved travoprost group only $35 \%(\mathrm{n}=07)$ experienced a decrease in OSDI score which could be due to reduction in IOP without any change in the category of OSDI severity. As seen in figure 5, BAK-free travoprost was well 
tolerated.

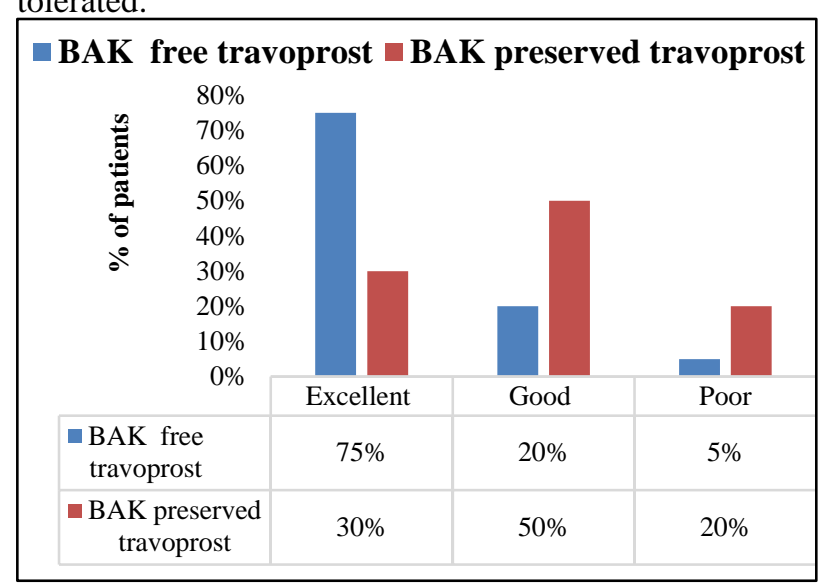

Figure 5: Global evaluation of tolerability in both the groups.

\section{Adverse drug reactions}

The conjunctival hyperaemia was encountered in $05(25 \%)$ patients in BAK-free travoprost group and in 08 (30\%) patients in BAK-preserved travoprost group followed by ocular irritation. There was no significant difference in occurrence of conjunctival hyperaemia between the study drugs $\left(\chi^{2}=0, \mathrm{df}=1, \mathrm{p}=1\right)$ although the percentage of patients with conjunctival hyperaemia was less in BAKfree travoprost group. None of the patients discontinued the study medication due to adverse drug reactions.

\section{DISCUSSION}

POAG is expected to be a significant contributing factor to the increasing rates of irreversible blindness. As the prevalence of this disease is expected to increase in the upcoming years, it is important that medical therapy be optimized to prevent the progression of the pathologic process and maximize the safety profiles to ensure patients' comfort. Medical treatment is effective if administered appropriately, but comfort concerns often cause patients to neglect taking their medications. Hyperaemia, pain, burning, and ocular discomfort are common causes of patient nonadherence and it has been found that nearly $70 \%$ of patients are non-adherent to their medications within the first year. Reduced comfort and consequent reduced adherence to dosing regimens are widely attributed to the preservative used in most glaucoma medications. BAK is repeatedly implicated in the ocular surface adverse events that are reported. ${ }^{12}$

Ocular surface disease is prevalent among patients with glaucoma, especially those who receive treatment with ophthalmic solutions. Although the exact mechanism for this remains unknown, BAK has been associated with a variety of ocular symptoms such as burning, stinging, foreign body sensation, and dry eye. BAK is a detergent used in ophthalmic solutions to prevent microbial growth and perpetuate medication distribution into the eye. However, it also disrupts cellular processes and reduces tear film production and stability. Eliminating or reducing exposure to BAK in ophthalmic solutions improves tear film stability, reduces conjunctival hyperemia, and improves dry eye symptoms. ${ }^{7,13-15}$

The present study was undertaken to examine the efficacy, safety, tolerability of BAK-free travoprost ophthalmic solution compared with BAK-preserved travoprost. The study demonstrated that the IOP-lowering efficacy was equivalent between BAK-free travoprost $0.004 \%$ and BAK-preserved travoprost $0.004 \%$. There was no significant difference with respect to conjunctival hyperaemia between the two study medications and BAKfree travoprost was well tolerated.

The results of the present study are consistent with results of the travoprost BAK-free regulatory trial, which demonstrated travoprost BAK-free was at least as efficacious as the BAK-preserved preparation of travoprost. Comparable outcome was observed in a study by Lewis $\mathrm{Ra}$ et al., which showed that travoprost BAKfree safely and effectively lowered IOP in eyes with openangle glaucoma. ${ }^{16}$

Furthermore a study by Gandolfi $\mathrm{S}$ et al, concluded that travoprost $0.004 \%$ without $\mathrm{BAK}$ is equivalent to travoprost $0.004 \%$ with BAK in both safety and efficacy. ${ }^{17}$

The study by Lopes JS et al, established that transitioning from BAK-containing latanoprost $0.005 \%$ to BAK-free travoprost $0.004 \%$ reduced IOP in patients with POAG or ocular hypertension who were intolerant to latanoprost. ${ }^{12}$ BAK-free travoprost $0.004 \%$ was a viable alternative for patients who require switching their IOP-lowering medications because of tolerability issues. In a literature review by Mirza SK et al., the author enumerates that BAK- free travoprost has demonstrated equal efficacy and less ocular surface toxicity than its preserved counterparts. ${ }^{9}$

In the above study BAK-free travoprost demonstrated significant decrease in OSDI scores. These findings are in line with results of the study by Henry JC et al, wherein travoprost BAK-free demonstrated improved mean OSDI scores, decreased hyperaemia and equal or better IOP control compared to either latanoprost or bimatoprost. ${ }^{8}$ In $70.2 \%$ of these patients, symptoms were reduced in severity by at least 1 level compared to results obtained in present study wherein $90 \%$ of patients experienced improvement in ocular surface disease symptoms. The improved comfort may be attributed to the absence of BAK in the travoprost preparation. Although conjunctival hyperaemia occurred less frequently in BAK-free travoprost group there was no statistical difference between both the groups. This is in line with the observations made in previous studies. . $^{8,9,18}$

The findings of this study have important implications for the medical management of patients with glaucoma, because OSD is common among glaucoma patients, and 
also because glaucoma patients have identified tolerability issues as barriers to compliance with IOP-lowering therapies. In choosing an IOP-lowering medication for glaucoma or ocular hypertension, both efficacy and tolerability should be considered. Given the far-reaching effects of ocular inflammation in regard to adherence and failure of filtration surgery, this advancement appears to be much needed. The IOP-lowering efficacy is equivalent between BAK-free travoprost $0.004 \%$ and BAK-preserved travoprost $0.004 \%$. Therefore, consider a switch from a BAK-preserved therapy to BAK-free travoprost $0.004 \%$ in patients with ongoing glaucoma who have comorbid OSD, or could consider initiating BAK-free travoprost $0.004 \%$ in new glaucoma patients who are at risk of developing OSD due to use of BAK-preserved products.

After an extensive search, to the best of our knowledge this is the first study comparing the efficacy, safety, tolerability of BAK free travoprost with BAK preserved travoprost amongst the Indian patients with POAG. The evaluations of the symptoms of ocular surface disease using a validated OSDI questionnaire adds strengths to the study. The present study did have few limitations as listed below:

It was open label study; sample size was calculated to evaluate a difference of $1 \mathrm{mmHg}$ between study medications but the difference obtained was small $(0.63$ $\mathrm{mmHg}$ ). To detect such a small difference a larger sample size is required. In addition, this study was not designed to address the long-term clinical outcomes of travoprost BAK-free therapy and objective tests were not used to evaluate symptoms of ocular surface disease. In future well designed randomised study with follow up for longer time period with a large sample size and using Schirmer test, Tear Breakup Time, corneal fluorescein stain is required to establish efficacy and safety of BAK-free travoprost over BAK-preserved travoprost.

BAK-free travoprost is an effective option for IOP reduction while avoiding $\mathrm{BAK}$ exposure in $\mathrm{POAG}$ patients with OSD.

\section{ACKNOWLEDGEMENTS}

We would like to thank the patients who participated in this study and also faculty of Department of Ophthalmology, Minto Ophthalmic hospital, and faculty of Department of Pharmacology Bangalore Medical College and Research Institute, Bengaluru for their extended support and immense help in this project.

Funding: No funding sources Conflict of interest: None declared

Ethical approval: The study was approved by the Institutional Ethics Committee

\section{REFERENCES}

1. Eyawo O, Nachega J, Lefebvre P, Meyer D, Rachlis $\mathrm{B}$, Lee CW, et al. Efficacy and safety of prostaglandin analogues in patients with predominantly primary open-angle glaucoma or ocular hypertension: a metaanalysis. Clin Ophthalmol. 2009;3:447-56.

2. Sambhara D, Aref AA. Glaucoma management: relative value and place in therapy of available drug treatments. Therapeutic Advances in Chronic Disease. 2014;5(1):30-43.

3. Sinha SK, Astbury N. Evaluation of the effectiveness of ophthalmic assistants as screeners for glaucoma in North India. Eye. 2011;25(10):1310-6.

4. Denis P, Covert D, Realini A. Travoprost in the management of open-angle glaucoma and ocular hypertension. Clinical ophthalmology. 2007;1(1):1124.

5. Kaštelan S, Tomić M, Metež Soldo K, SalopekRabatić J. How ocular surface disease impacts the glaucoma treatment outcome. BioMed research international. 2013 Oct 9;2013.

6. Yuksel N. Evaluation of Ocular Surface Disease Associated with Glaucoma Patients. European Ophthalmic Review. 2013;7(2):81-3.

7. Cvenkel B, Stunf S, Kirbis SI, Flezar SM. Symptoms and signs of ocular surface disease related to topical medication in patients with glaucoma. Clinical Ophthalmology. 2015;9:625-31.

8. Henry JC, Peace JH, Stewart JA, Stewart WC. Efficacy, safety, and improved tolerability of travoprost BAK-free ophthalmic solution compared with prior prostaglandin therapy. Clinical ophthalmology. 2008;2(3):613-21.

9. Mirza, Sophia K, Johnson SM. Efficacy and Patient Tolerability of Travoprost BAK-Free Solution in Patients with Open-Angle Glaucoma and Ocular Hypertension. Clinical Ophthalmology. 2010:877-88.

10. Fechtner RD, Godfrey DG, Budenz D, Stewart JA, Stewart WC, Jasek MC. Prevalence of ocular surface complaints in patients with glaucoma using topical intraocular pressure-lowering medications. Cornea. 2010;29:618-21.

11. Walt J, Rowe M, Stern K. Evaluating the functional impact of dry eye: The Ocular Surface Disease Index. Drug Inf J. 1997;31:1436

12. Lopes JF, Hubatsch DA, Amaris P. Effect of benzalkonium chloride-free travoprost on intraocular pressure and ocular surface symptoms in patients with glaucoma previously on latanoprost: an open-label study. BMC Ophthalmology. 2015;15:166.

13. Herreras JM, Pastor JC, Calonge M, Asensio VM. Ocular surface alteration after long-term treatment with an antiglaucomatous drug. Ophthalmology. 1992;99:1082-8.

14. Turacli E, Budak K, Kaur A, Mizrak B, Ekinci C. The effects of long-term topical glaucoma medication on conjunctival impression cytology. Int Ophthalmol. 1997;21:27-33.

15. Kaur IP, Lal S, Rana C, Kakkar S, Singh H. Ocular preservatives: associated risks and newer options. Cutan Ocul Toxicol. 2009;28(3):93-103.

16. Lewis RA, Katz GJ, Weiss MJ, Landry TA, Dickerson JE, James JE, et al. Travoprost $0.004 \%$ with and 
without benzalkonium chloride: a comparison of safety and efficacy. J Glaucoma. 2007;16(1):98-103.

17. Gandolfi S, Paredes T, Goldberg I, Coote M, Wells A, Volksone L, et al. Comparison of a travoprost BAKfree formulation preserved with polyquaternium-1 with BAK-preserved travoprost in ocular hypertension or open-angle glaucoma. Eur J Ophthalmol. 2012 JanFeb;22(1):34-44.

18. Aihara M, Otani S, Kozaki J, Unoki K, Takeuchi M, Minami K, et al. Long-term effect of BAK-free travoprost on ocular surface and intraocular pressure in glaucoma patients after transition from latanoprost. J Glaucoma. 2012;21(1):60-4.

Cite this article as: Jayanthi CR, Divyashree RN, Sujatha BL. Efficacy and safety of topical BAK-free travoprost $0.004 \%$ versus BAK-preserved travoprost $0.004 \%$ in the treatment of primary open angle glaucoma: a comparative study at a tertiary care hospital. Int J Basic Clin Pharmacol 2017;6:2199205. 